\title{
NMR and Molecular Modelling Studies of the Binding of Amicetin Antibiotic to Conserved Secondary Structural Motifs of 23S Ribosomal RNAs
}

\author{
James Donarski, Christos Shammas, Ryan Banks, Vasudevan Ramesh
}

Received: February 2, 2006 / Accepted: March 6, 2006

(C) Japan Antibiotics Research Association

\begin{abstract}
The interaction of a highly conserved secondary structural RNA motif of Halobacterium halobium and Escherichia coli $23 \mathrm{~S}$ ribosomal RNAs with the peptidyl transferase inhibitor antibiotic amicetin has been investigated by proton NMR spectroscopy and molecular modelling. The NMR spectra of the synthetic 35mer RNA motifs revealed spectral features characteristic of a stable, well folded A-RNA type tertiary conformation, including resolved resonances assigned to unpaired bases located in the middle of the motif strongly implicated in amicetin binding. Addition of amicetin to the 35mer RNA samples was accompanied by significant and discrete changes to the spectra which can be qualitatively interpreted to the changes induced to the local conformation of the RNA motifs arising from the formation of a specific complex with amicetin. These results are also supported by the unconstrained molecular model of RNAamicetin complex which highlights potential interactions between the two molecular components.
\end{abstract}

Keywords Halobacterium halobium 23S ribosomal RNA, Escherichia coli 23S ribosomal RNA, peptidyl transferase centre, amicetin, NMR spectroscopy, CD spectroscopy, molecular modelling

\section{Introduction}

Interest in the involvement of RNA in protein biosynthesis has increased following extensive studies on the binding of antibiotic drugs to specific target sites on ribosomal RNA
(rRNA) [1-3]. It is noteworthy that resistance to antibiotics often involves a single mutation $[4,5]$ or methylation of a specific nucleotide in a highly conserved structural motif of rRNA [6], confirming the highly specific nature of RNAantibiotic interactions. Recently, the crystal structures of the large 50S subunit [7] and 30S small subunit [8] of ribosomal RNA and their complexes with a number of antibiotics $[9,10]$ have been solved.

The most important functional site on the ribosome is the 'peptidyl transfer centre [3,11] and previous resistance studies using a number of antibiotics such as chloramphenicol and 'Macrolide, Lincosamide and Streptogramin' (MLS) antibiotics have located this within 23S like rRNAs in the highly conserved central circle of domain V (Fig. 1a) $[4,5]$. However, based on more recent experiments, it has been revealed that antibiotic inhibition of peptide transfer can also take place by other mechanisms, attributing functional and structural roles for analogous conserved structural motifs lying adjacent to the catalytic transfer centre $[1,12]$. One such antibiotic, amicetin, belongs to a functionally related family of drugs which share a hexosecytosine moiety (Fig. 1b) [2, 13]. Amicetin has been described as an 'universal' antibiotic as it binds to a highly conserved structural motif of 23S like rRNAs which occur in organisms of different evolutionary origins (archae, bacteria and eukarya) [12]. The secondary structures of the highly conserved rRNA motifs of $H h$ and $E$. coli within the central circle domain $\mathrm{V}$, with affinity for amicetin, are illustrated in Figure 1c, d.

The precise inhibitory role of amicetin in protein synthesis remains unclear at this stage. It has been
V. Ramesh (Corresponding author), J. Donarski, C. Shammas, R. Banks: School of Chemistry, The University of Manchester,
Sackville Street, P.O. Box 88, Manchester, M60 1QD, United Kingdom, E-mail: vasudevan.ramesh@manchester.ac.uk 
postulated that whereas some drugs belonging to the category of chloramphenicol and macrolide antibiotics may impair initial binding of the aminoacyl tRNA in the A-site, other hexose-cytosine drugs such as amicetin may block the movement of the aminoacyl group towards the P-site bound peptidyl tRNA and that the drug binding sites lie very close to the catalytic centre $[4,12]$. Previously, amicetin binding to $23 \mathrm{~S}$ rRNA of intact $H h$ ribosomes were probed by chemical footprinting experiments using nucleotide specific chemical modification reagents in order to establish the amicetin binding site [12]. However, these experiments failed to yield discernible footprints and hence exact details regarding the site and mode of amicetin binding to $\mathrm{Hh}$ rRNA have remained obscure, despite the generation of a spontaneous single nucleotide amicetin-resistant mutant (U2457 $\rightarrow$ C) strongly implying amicetin binding [12] Similar "non-footprinting" antibiotics such as sparsomycin (most potent inhibitor of protein synthesis) involved in ribosomal activity, causing a specific resistance mutation of $H h$ 23S rRNA have been reported [14]. It is thus quite likely that the non-footprinting antibiotics like amicetin, sparsomycin interact with the $H h$ RNA to exert their effect on the activity of ribosome without necessarily protecting the bases from chemical modification. Clearly, more precise three dimensional structural studies are required at the molecular level if we are to begin to understand the mode of interaction of rRNA motifs with antibiotics in mechanistic detail.

In this paper, we describe the successful application of NMR and unconstrained molecular modelling to characterise the synthetic 35mer RNAs corresponding to the highly conserved secondary structural RNA motifs of $H h$ and E. coli $23 \mathrm{~S}$ rRNAs shown to have affinity for amicetin. We provide direct evidence for the interaction between the RNA motifs and amicetin. There is increasing evidence that smaller synthetic oligonucleotide analogues of the well conserved rRNA secondary structural motifs can adopt the relevant three dimensional fold and function autonomously [15]. Determining the structures of such motifs will be an essential step in understanding the specificity of RNA-drug or RNA-protein interactions at the molecular level [16].

\section{Materials and Methods}

\section{Antibiotic}

The amicetin antibiotic used for RNA binding studies was obtained as a gift from Pharmacia-Upjohn, USA.

\section{Chemical Synthesis of RNA}

The single-stranded 35mer RNA samples were synthesised by automated solid-phase synthesis and purified by HPLC procedures (Oswel, U.K.). The purity of the samples was checked by capillary zone electrophoresis which revealed a single sharp peak, they also showed good stability with a high $T_{\mathrm{m}}$ of $75^{\circ} \mathrm{C}$. The $35 \mathrm{mer}$ RNAs used in this study correspond to the sequences of the motifs shown in Figure 1c, d i.e., Hh RNA, 14mer 5'-C2089-U2102-3'/6mer linker $5^{\prime}$-CUUCGG-3'/15mer $5^{\prime}$-A2452-G2466-3' and E. coli RNA, 14mer 5'-C2063-U2076-3'/6mer linker 5'CUUCGG-3'/15mer $5^{\prime}$-A2433-G2447-3'. Prior to spectral characterization, the samples were heated to $70^{\circ} \mathrm{C}$ for 2 minutes and slowly cooled to room temperature to ensure that the RNA molecules adopted a homogeneous, properly folded conformation, free of any aggregation.

\section{Empirical Free Energy Calculation}

The empirical free energy calculation of the 35mer RNA motifs was carried out using the Mfold program [17] available on the web server (http://www.bioinfo.rpi.edu/ applications/mfold).

\section{Spectroscopy}

The CD spectra were measured on a Jasco-J720 spectropolarimeter at $295 \mathrm{~K}$ using a quartz cell with a $1 \mathrm{~cm}$ path length and scanning the UV spectral region from 220 to $320 \mathrm{~nm}$. Baseline error due to cell and buffer was subtracted from each spectrum before plotting the $\mathrm{CD}$ spectra of the individual RNA samples.

\section{NMR Spectroscopy}

One- and two-dimensional proton NMR experiments [18] were carried out using Varian Unity $500 \mathrm{MHz}$ and Varian Inova $600 \mathrm{MHz}$ NMR spectrometers, equipped with a triple resonance inverse geometry gradient probe and electronic variable temperature unit. The spectra were acquired using Vnmr 6.1C software hosted by a Sun UNIX workstation and the manufacturer supplied pulse programs with modifications where necessary. The spectral width was set to $22 \mathrm{ppm}$ for experiments in $\mathrm{H}_{2} \mathrm{O}$ and the transmitter/ carrier was positioned on the water signal to minimise any artefacts. The large resonance due to the water protons was suppressed by the WATERGATE pulse sequence [19]. Typically, 128 scans were averaged for each FID before apodisation and Fourier transformation. ${ }^{1} \mathrm{H}$ chemical shifts are reported with respect to dilute concentration of internal dioxane $(0.01 \% \mathrm{v} / \mathrm{v})$ used as an internal reference standard ( $\delta 3.761 \mathrm{ppm})$ [18].

Most of the 1D and 2D NMR data were initially processed using Varian Vnmr 6.1C software. For display 
and analysis purposes, the data were later reprocessed using both MestRe-C 3.4 (http://www.mestrec.com) running on Microsoft Windows XP and NMRPipe software (http://www.spin.niddk.nih.gov/bax/software/NMRPipe running on the RedHat Linux 9.0 operating system (http://www.redhat.com). Usually, each time domain $\left(t_{1}, t_{2}\right)$ data was zero-filled twice before apodisation by a shifted sine bell function and then Fourier transformed to yield frequency domain $\left(\delta_{1}, \delta_{2}\right)$ spectra.

The NMR samples, as a $0.6 \mathrm{ml}$ solution each, were prepared by dissolving the RNA $(0.60 \mathrm{mM})$ in $\mathrm{H}_{2} \mathrm{O} / \mathrm{D}_{2} \mathrm{O}$ $(9: 1)$ solvent mixture containing $20 \mathrm{mM} \mathrm{PO}_{4}^{3-}$, pH 6.2 . Titrations with amicetin antibiotic were carried out by adding microlitre volumes of a stock solution of the antibiotic to RNA samples.

\section{Unconstrained Molecular Modelling and Molecular Dynamics Simulation [20, 21]}

\section{Modelling of Amicetin}

The amicetin model was constructed within HyperChem 7.5. Charge values for individual atoms of amicetin were determined using $a b$ initio calculations with the medium sized $6-31 \mathrm{G}^{*}$ basis set. The antibiotic starting coordinates were then exported into X-PLOR and energy minimized. The minimized structure was then subjected to a $1 \mathrm{~ns}$ molecular dynamics (MD) simulation in vacuo at $300 \mathrm{~K}$, where at every $100 \mathrm{ps}$ the model was cooled and its conformation recorded.

\section{Modelling of 35mer $H h$ RNA Motif}

The spatial coordinates of the $H h$ 35mer RNA were derived from the $2.4 \AA$ crystal structure of the large 50S ribosomal subunit from Haloarcula marismortui (PDB accession number 1FFK) [7]. The latter showed a high degree of sequence homology to the $H h 35$ mer motif, including in the critical dinucleotide and mononucleotide bulge regions of the motif. Specifically, mutation of nucleotides at 2095 (GA) and $2461(\mathrm{C}-\mathrm{U})$ were made to generate the respective Hh RNA primary sequence. The UUCG tetra loop was constructed with the same initial coordinates as that given for the $2.8 \AA$ resolution structure (PDB accession number 1F7Y) [22]. The resulting 35mer RNA motif (Fig. 1c) was then geometry optimized using X-PLOR. NOE energy terms were then incorporated into the forcefield to maintain Watson-Crick base pairs as predicted by the secondary structural model, and confirmed by ${ }^{1} \mathrm{H}$ imino-imino sequential assignment. NOE contacts of $2.3 \pm 0.4 \AA$ were applied between the atomic groups maintaining the canonical G-C and A-U Watson-Crick base pairs. The 35 mer model was then subjected to a 60 ps MD simulation at $300 \mathrm{~K}$ in vacuo, followed by a cooling step and energy minimization.

Docking Model of Amicetin-Hh 35mer RNA Complex

The above energy minimised structural models of amicetin and $H h$ 35mer RNA were placed $20 \AA$ apart within HyperChem 7.5 and the new coordinate file was then transferred back into X-PLOR. Simulated annealing of the amicetin-RNA complex began with a 40000 step energy minimization. Energy terms within the forcefield included bond length, angle, dihedral, van der Waals, improper, Hbond, and also an NOE energy term.

$$
\mathrm{E}_{\mathrm{TOTAL}}=\sum \mathrm{E}_{\text {bond }}+\mathrm{E}_{\text {ang }}+\mathrm{E}_{\text {dihe }}+\mathrm{E}_{\mathrm{vdw}}+\mathrm{E}_{\text {impr }}+\mathrm{E}_{\mathrm{Hbon}}+\mathrm{E}_{\mathrm{NOE}}
$$

The latter energy term was applied to maintain the base pairing scheme implied by the proposed secondary structural model and the observed ${ }^{1} \mathrm{H}$ imino-imino NOE sequential connectivities. For the maintenance of the Watson-Crick base pairing schemes, inter-proton distance constraints of $2.3 \pm 0.4 \AA$ were applied to each H-bond of the canonical G-C and A-U base pairing schemes. Force constants for all bond lengths, bond angles, improper angles and dihedral angles were set at 1000, 500, 500 and $500 \mathrm{kcal} \mathrm{mol}^{-1}$, respectively. The high dihedral force constant ensured purine rings remained parallel, whilst the high improper force constant helped maintain perfect chirality. Following energy minimization, the docking model was subjected to a MD simulation at $300 \mathrm{~K}$ followed by a cooling step. Representative conformations were recorded after every $100 \mathrm{ps}$ during alns simulation.

\section{Results and Discussion}

\section{Design, Synthesis and Stability of Single-Stranded 35mer RNAs for Binding to Amicetin}

We selected the highly conserved secondary structural RNA motifs corresponding to $H h$ and E. coli $23 \mathrm{~S}$ rRNA, the helical stem motif in the upper part of the central circle region (Fig. 1a), for amicetin binding based on the results previously reported in the literature [12] and discussed above. The underlined nucleotides of the hexanucleotide linker ( $5^{\prime}$-CUUCGG-3'), occuring commonly in ribosomal RNAs, has been shown to form a stable tetraloop and confer high thermal stability [23]. The empirical free energy calculations showed that the synthetic 35 mer motif, despite the UUCG tetra loop and internal bulges (Fig. 1c, d), has a low free energy $\left(H h-16.0 \mathrm{kcal} \mathrm{mol}^{-1}\right.$ and $E$. coli $-18.0 \mathrm{kcal} \mathrm{mol}^{-1}$ ) and hence is predicted to be endowed with good stability. These values correlate well 


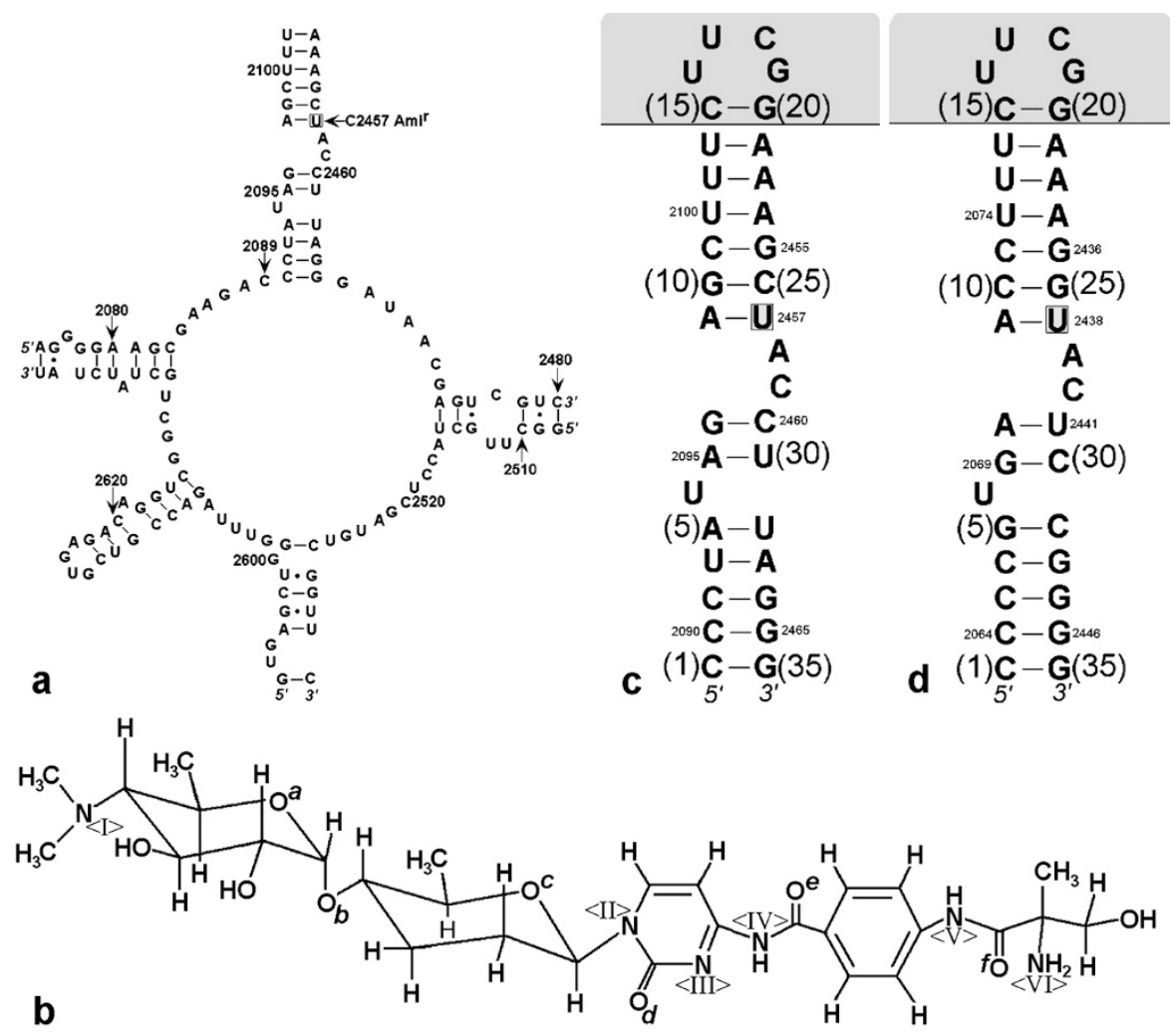

Fig. 1 (a) A schematic illustration of the secondary structural model of the peptidyl transferase central circle region of domain $\mathrm{V}$. The four digit numbering signifies the numbering scheme used for the Hh 23S rRNA sequence. The helical stem region above the central circle shows the site of amicetin induced mutation, C2457 Amir (b) the chemical structure of the peptidyl transferase inhibitor antibiotic amicetin. Labels $b, d$ and $<111>$ indicate sites of interaction with 35mer RNA motif in the molecular model of the complex. (c) the highly conserved amicetin binding 35mer RNA motif of Hh $23 \mathrm{~S}$ rRNA spanning the sequence 14 mer 5'-C2089-U2102-3'/6mer linker 5'-CUUCGG-3'/15mer 5'-A2452-G2466-3' (d) the analogous 35mer RNA motif of the E. coli 23S rRNA spanning the sequence 14mer 5'-C2063-U2076-3'/6mer linker 5'-CUUCGG-3'/15mer 5'A2433-G2447-3' is shown alongside.

with the data noted for an analogous 38mer RNA from EMCV IRES, endowed with a GNRA tetraloop, an internal $7 \mathrm{nt}$ asymmetrical bulge and a single $\mathrm{C}-\mathrm{U}$ mismatch, which showed a predicted free energy of $-14.0 \mathrm{kcal} \mathrm{mol}^{-1}$ and demonstrated to produce good NMR spectra [15].

CD spectroscopy is a useful and rapid method to identify experimental conditions before commencing an NMR structural investigation. DNA and RNA exhibit readily distinguishable $\mathrm{CD}$ spectral features correlating with their B-DNA and A-RNA type conformation respectively, with characterisic ellipticity bands in the UV region $(190 \sim 320 \mathrm{~nm})$ [24]. We measured the CD spectra of the binding of 2 molar equivalents of amicetin to $E$. coli $35 \mathrm{mer}$ RNA and analysis of the spectra showed a large change with a decrease in intensity of the $280 \mathrm{~nm}$ band, noted to be sensitive to conformational variation taking place within the A-form helix [24]. These results demonstrate that the synthetic 35 mer RNA motif is endowed with a well folded
A-RNA type conformation with the disposition of a binding site for amicetin.

\section{NMR Assignment of Imino Protons and Titration with Amicetin}

The ${ }^{1} \mathrm{H}-\mathrm{NMR}$ spectra of synthetic $H h$ and $E$. coli $35 \mathrm{mer}$ RNAs were measured both in $\mathrm{H}_{2} \mathrm{O}$ and $\mathrm{D}_{2} \mathrm{O}$ which remained invariant over prolonged periods thus attesting to their stability [25]. The spectra showed resonances due to non-exchangeable protons in the characteristic narrow chemical shift $8.5 \sim 3.5 \mathrm{ppm}$ range and several shifted resonances in the low-field end of the spectrum $15.0 \sim 11.5 \mathrm{ppm}$ arising from the exchange labile imino protons of the hydrogen-bonded $\mathrm{C}-\mathrm{G}$ and A-U basepairs making up the helical secondary structure of the RNA motif. As an illustration, the $600 \mathrm{MHz}$ proton NMR spectrum of $H h 35$ mer RNA measured in $\mathrm{H}_{2} \mathrm{O}$ at $283 \mathrm{~K}$ is shown in Figure $3 \mathrm{a}$ where the above mentioned resonances 
due to labile imino protons can be identified. After taking into account the observed overlap, the number of resonances in the $15 \sim 12 \mathrm{ppm}$ correspond well with the estimated number of hydrogen bonded A-U and C-G base pairs stemming from the predicted helical secondary structure of the $H h$ RNA motif shown in Figure 1c. In addition, the observation of several resolved resonances in the $11.5 \sim 9.5 \mathrm{ppm}$ region is usually associated with non hydrogen bonded, labile imino protons of $U$ and $G$ bases [26] and hence they may be reliably identified with the unpaired bases belonging both to the highly conserved bulge(s) in the middle and the capped nucleotide loop linker at the top of the predicted motif (Fig. 1c). Similarly, the spectrum of E. coli 35mer RNA was measured in $\mathrm{H}_{2} \mathrm{O}$ with analogous pattern of resonances (not shown) to the $H h$.

Figure 2 shows the NOESY spectrum of uncomplexed $H h$ 35mer RNA in $\mathrm{H}_{2} \mathrm{O}$, where sequential connectivities have been traced for imino protons in close proximity of nucleotides adjacent to each other in the sequence of the stem-loop. All of the cross peaks have been unambiguously identified and their connectivities assigned to sequencespecific nucleotides of the stem-loop. A striking feature of the spectrum is the identification of NOESY cross peak between the imino protons of U30 and G8.

The chemical shifts of the nucleotides U16 (12.36 ppm) and G19 (9.71 ppm) of the UUCG tetra loop correlate well with those found in other similarly sized RNA motifs containing the same UUCG tetra loop [27].

After the addition of increasing molar equivalents of amicetin to the $H h 35$ mer RNA sample, a variety of changes in chemical shift and selective line broadening to the assigned imino proton resonances can be observed in the spectrum of the complex measured in $\mathrm{H}_{2} \mathrm{O}$ (Fig. 3a-e). Thus, amicetin induced chemical shift changes to some of the imino proton resonances of hydrogen-bonded basepairs (C-G and A-U) and selective broadening of the resolved resonances of the non hydrogen bonded imino protons of $\mathrm{G}$ and $U$ bases are indicated by vertical lines. It is noteworthy that resolved resonances due to nucleotides U6, G8, U30, U31 located in the bulge region of the motif (Fig. 1c) exhibit selective broadening upon binding to amicetin. As indicated, progressive line broadening is also caused to the mutationally sensitive residue U26 during the same titration. However, it may also be noted that the resonances of the loop nucleotides U16, U17, G19 remain unaffected throughout the titration.

NMR titrations of $H h$ and $E$. coli 35-mer RNAs with amicetin were also carried out in $\mathrm{D}_{2} \mathrm{O}$ in order to monitor changes induced to the non-exchangeable proton resonances of the respective RNAs after progressive

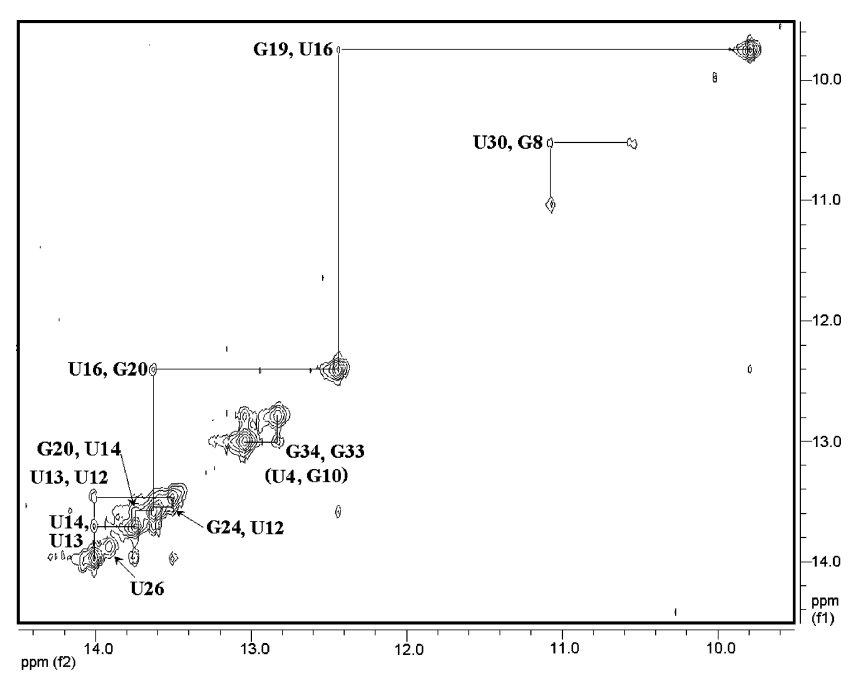

Fig. $2500 \mathrm{MHz}{ }^{1} \mathrm{H}-{ }^{1} \mathrm{H}$ correlated, phase-sensitive NOESY spectrum of $\mathrm{Hh} 35 \mathrm{mer}$ RNA $(0.60 \mathrm{mM})$ dissolved in $\mathrm{H}_{2} \mathrm{O} / \mathrm{D}_{2} \mathrm{O}(9: 1), 20 \mathrm{mM} \mathrm{PO}_{4}^{3-}, \mathrm{pH} 6.2$ at $283 \mathrm{~K}$.

Mixing time used for cross relaxation between protons in close proximity was $250 \mathrm{~ms}$. Connectivities tracing cross peaks due to cross relaxation between adjacent imino protons $\left(\mathrm{NH}_{\mathrm{i}}-\mathrm{NH}_{\mathrm{i}+1}\right)$ of each base pair are labelled sequence specifically.

addition of the antibiotic (not shown). Again amicetin induced selective broadening to some of the resolved RNA resonances was observed suggesting a specific interaction taking place between the aromatic protons of the RNA and amicetin.

It is perhaps likely that the broadening of the specific resonances noted above (Fig. 3a-e) arises from exchange effects [28]. One possibility is that the exchange process involved is simply between the unbound RNA and RNAamicetin complex. However, in the resonances of hydrogen bonded $\mathrm{C}-\mathrm{G}$ and $\mathrm{A}-\mathrm{U}$ basepairs, for whom the above chemical shift changes occur, there is no apparent correlation between shift change and the linewidth. An alternative possibility is that there are exchange processes which specifically involve the non-hydrogen bonded imino protons of unpaired $\mathrm{G}$ and $\mathrm{U}$ bases located in the flexible regions of the RNA motif (such as the bulge in the middle) and further detailed studies are required to identify the specific motions responsible for the observed line broadening. This phenomenon is paralleled by the selective line-broadening observed for the backbone peptide $\mathrm{NH}$ protons in the spectra of selectively labelled ${ }^{15} \mathrm{~N}$-trp repressor on binding to the operator oligonucleotide [29]. Here the broadening selectively affects residues, most notably, in the flexible DNA-binding helix-turn-helix domain. These selective changes in linewidth imply the existence of some dynamic process(es) within the RNA- 


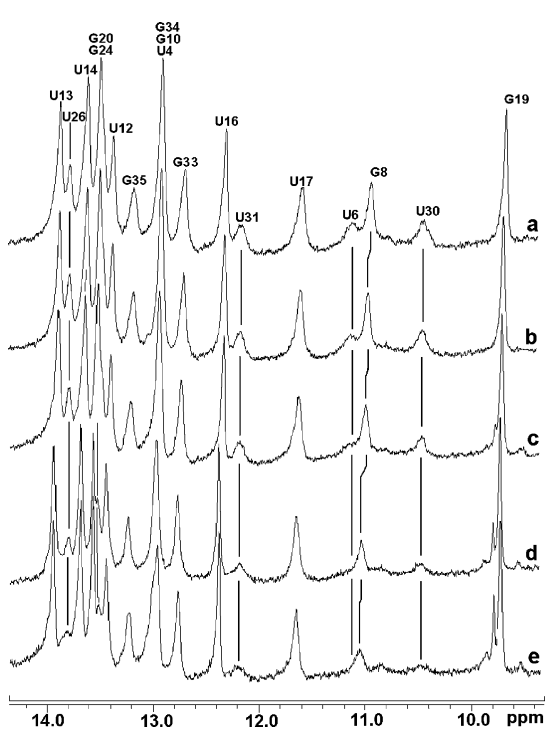

Fig. $3600 \mathrm{MHz}{ }^{1} \mathrm{H}-\mathrm{NMR}$ spectra of the titration of synthetic Hh 35mer RNA $(0.60 \mathrm{mM})$ in $\mathrm{H}_{2} \mathrm{O}_{2} \mathrm{D}_{2} \mathrm{O}(9: 1)$ containing $20 \mathrm{mM} \mathrm{PO}_{4}{ }^{3-}, \mathrm{pH} 6.2$ at $283 \mathrm{~K}$ with amicetin antibiotic (in molar equivalents) (a) 0.0 , (b) 0.18 , (c) 0.70 , (d) 1.66, (e) 2.75 .

Chemical shift changes and selective broadening of resonances are indicated by vertical lines.

amicetin complex, affecting those bases close to the site of interaction.

\section{Unconstrained Molecular Modelling of Amicetin-RNA Complex}

The unconstrained model of amicetin-RNA complex highlights several key interactions between the two molecular components which may be functionally significant and a section of the model is shown in Figure 4. The imino proton $\mathrm{H} 3$ of the pyrimidine ring of the bulged residue U6 (2094) makes a single hydrogen bond with the carbonyl oxygen, labelled as d, of the aromatic cytosine ring of the antibiotic. Similarly, the ribose $2{ }^{\prime}-\mathrm{OH}$ donor group of the same residue U6 (2094) makes a hydrogen bond with the glycosidic-O-linker, labelled as b. The model also predicts some kind of electrostatic interaction arising between the antibiotic tertiary amide $<$ III $>$ of cytosine ring and the carbonyl oxygen $\mathrm{O} 4$ of the pyrimidine ring of U6 (2094), since the two atoms are only $2.9 \AA$ apart.

The unconstrained model of the complex shows a folded conformation for the amicetin antibiotic. This folding appears strained, but its stability is gained from a network of intra-molecular hydrogen bonds contacts and stacking interaction (Fig. 4). The proposed folded conformation, which consists of exposed potential hydrogen bond donors and acceptors across the saccharide and peptide regions,

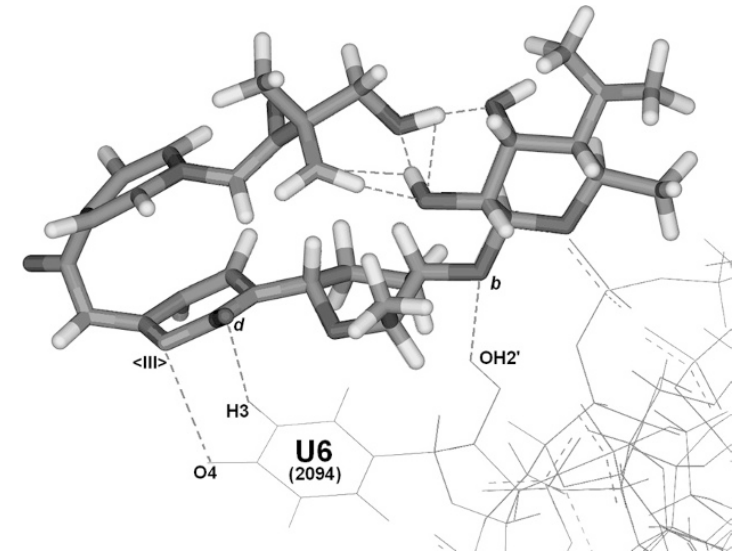

Fig. 4 Section of the unconstrained molecular model of amicetin-Hh 35mer RNA complex after energy minimisation.

The model highlights intra- and intermolecular hydrogen bond interactions $(2.3 \pm 0.4 \AA)$ within the complex as shown by dotted lines.

may be structurally critical for RNA binding.

\section{Conclusion}

In conclusion, we have successfully designed and chemically synthesised two 35 mer ribosomal RNA motifs of $H h$ and E. coli $23 \mathrm{~S}$ rRNAs and demonstrated by NMR spectroscopic measurements that they adopt a stable, well folded A-RNA conformation in solution. The results of the NMR studies also provide direct experimental evidence for the specific binding of amicetin antibiotic to the RNA motifs and this is supported by the unconstrained molecular modelling of the amicetin-RNA complex. The above results lay a strong foundation for further NMR studies to determine the $3 \mathrm{D}$ structure of the $35 \mathrm{mer}$ RNA motifs complexed to amicetin and the amicetin-resistant mutants. These studies should facilitate the determination of factors governing the specificity of interaction between the two molecular components (RNA and amicetin) from a threedimensional structural standpoint. In a wider perspective, the proposed NMR studies will provide a deeper and critical insight into the molecular basis of antibiotic action and antibiotic resistance [30].

Acknowledgements We gratefully acknowledge The Wellcome Trust, London (U.K.) and EPSRC for providing funds to carry out this project. We thank the staff of the MRC Biomedical NMR Centre, National Institute for Medical Research, Mill Hill, London for their valuable assistance and support. We thank Marie Phelan for her comments on the manuscript. 
References

1. Mankin AS. Ribosomal antibiotics. Mol Biol 35: 509-520 (2001)

2. Vazquez D. Inhibitors of Protein Biosynthesis. SpringerVerlag, Berlin (1979)

3. Hermann T. Chemical and functional diversity of small molecule ligands for RNA. Biopolymers 70: 4-18 (2003)

4. Kirillov S, Porse BT, Vester B, Wooley P, Garrett RA. Movement of the $3^{\prime}$-end of tRNA through the peptidyl transferase centre and its inhibition by antibiotics. FEBS Lett 406: 223-233 (1997)

5. Vester B, Garrett RA. The importance of highly conserved nucleotides in the binding region of chlorampenicol at the peptidyl transfer center of Escherichia coli $23 \mathrm{~S}$ ribosomal RNA. EMBO J 7: 3577-3587 (1988)

6. Cundliffe E. Involvement of specific portions of rRNA in defined ribosomal functions: a study utilizing antibiotics. In Structure, Function and Genetics of Ribosomes. Ed., B. Hardesty et al., pp. 586-604, Springer-Verlag, New York (1986)

7. Ban N, Nissen P, Hansen J, Moore PB, Steitz TA. The complete atomic structure of the large ribosomal subunit at 2.4 A resolution. Science 289: 905-920 (2000)

8. Carter AP, Clemens WM, Brodersen DE, Morgan-Warren RE, Wimberley B, Ramakrishnan V. Functional insights from the structure of the $30 \mathrm{~S}$ ribosomal subunit and its interactions with antibiotics. Nature 407: 340-348 (2000)

9. Schlunzen F, Zarivach R, Harms J, Bashan A, Tocil A, Albercht R, Yonath A, Franceshi F. Structural basis for the interaction of antibiotics with peptidyl transferase center in eubacteria. Nature 413: 814-818 (2001)

10. Hansen J, Moore PB, Steitz TA. Structures of five antibiotics bound at the peptidyl transferase centre of the large ribosomal subunit. J Mol Biol 330: 1061-1075 (2003)

11. Polacek N, Mankin AS. The ribosomal peptidyl transferase center: structure, function, evolution, inhibition. Cri Rev in Biochem Mol Biol 40: 285-311 (2005)

12. Leviev IG, Fonseca CR, Phan H, Garrett RA, Heilek G, Noller HF, Mankin AS. A conserved secondary structural motif in the 23S rRNA defines the site of interaction of amicetin, a universal inhibitor of peptide formation. EMBO J 13: 1682-1686 (1994)

13. Smith JL, Sundaralingam M. The structure of the antibiotic amicetin consisting of nucleobase, disaccharide and amino acid moieties. Acta Cryst B37: 1095-1101 (1981)

14. Tan GT, DeBlasio A, Mankin AS. Mutations in the peptidyl transferase centre of 23S rRNA reveal the site of action of sparsomycin, a universal inhibitor of translation. J Mol Biol 261: 222-230 (1996)

15. Phelan M, Banks RJ, Conn GL, Ramesh V. NMR studies of the structure and $\mathrm{Mg}^{2+}$ binding properties of a conserved 'hammerhead' RNA motif of EMCV picornavirus RNA. Nucleic Acids Res 32: 4715-4724 (2004)

16. Gallego J, Varani G. Targeting RNA with small-molecule drugs: therapeutic promise and chemical challenges. Acc Chem Res 34: 836-884 (2001)

17. Zuker M. Mfold web server for nucleic acid folding and hybridization prediction. Nucleic Acids Res 31: 3406-3415 (2003)

18. Wuthrich K. NMR of Proteins and Nucleic Acids. John Wiley \& Sons USA, New York (1986)

19. Piotto M, Saudek V, Sklenar V. Gradient-tailored excitation for single-quantum NMR spectroscopy of aqueous solutions. J Biomol NMR 2: 661-665 (1992)

20. Brunger AT. X-PLOR Version 3.1 Manual. Yale University Press New Haven USA, Connecticut (1992)

21. Miller JL, Cheatham TE, Kollman PA. Simulation of nucleic acids. In Oxford Handbook of Nucleic Acids. Ed., S. Neidle, pp. 99-115 Oxford University Press, USA (1999)

22. Ennifar E, Nikulin A, Tishchenko S, Serganov A, Nevskaya N, Garber M, Ehresmann B, Ehresmann C, Nikonov S, Dumas P. The crystal structure of UUCG tetraloop. J Mol Biol 304: 35-42 (2000)

23. Varani G, Cheong C, Tinoco I. Structure of an unusually stable RNA hairpin. Biochemistry 30: 3280-3289 (1991)

24. Gray DM, Huang SH, Johnson KH. Absorption and circular dichroism of nucleic acid duplexes and triplexes. Methods Enzymol 261: 271-299 (1995)

25. Donarski JA. Synthesis and NMR studies of the binding of amicetin antibiotic to conserved secondary structural RNA motifs of $23 \mathrm{~S}$ ribosomal RNAs. Ph. D. Thesis, UMIST (2004)

26. Varani G, Aboul-ela F, Allain FHT. NMR investigation of RNA structure. Progress in NMR spectroscopy 29: 51-127 (1996)

27. Jiang F, Fiala R, Live D, Kumar A, Pate DJ. RNA folding topology and intermolecular contacts in the AMP-RNA aptamer complex. Biochemistry 35: 13250-13266 (1996)

28. Bocharov EV, Gudkov AT, Budovskaya EV, Arseniev AS. Conformational independence of $\mathrm{N}$ - and $\mathrm{C}$-domains in ribosomal protein L7/L12 and in the complex with protein L10. FEBS Lett 423: 347-350 (1988)

29. Ramesh V, Frederick RO, Syed SEH, Gibson CF, Yang JC, Roberts GCK. The interactions of Escherichia coli trp repressor with tryptophan and with an operator oligonucleotide. NMR studies using selective ${ }^{15} \mathrm{~N}$-labelled protein. Eur J Biochem 225: 601-608 (1994)

30. Levy SB. Antibiotic Resistance: An Ecological Imbalance. Abstracts of papers of Symp on Antibiotic resistance: origins, evolution, selection and spread Ciba Foundation, No. 207, London (1996) 